\title{
Bond Characterization by Detection and Manipulation of Particle Mobility in an Optical Evanescent Field Biosensor
}

\author{
Kim van Ommering ${ }^{1,2}$, Marjo Koets ${ }^{1}$, Rik Paesen ${ }^{2}$, Leo $\mathbf{J}$ van IJzendoorn $^{2}$ and \\ Menno W J Prins ${ }^{1,2}$ \\ ${ }^{1}$ Philips Research Laboratories, 5656 AE Eindhoven, The Netherlands \\ ${ }^{2}$ Department of Applied Physics, Eindhoven University of Technology, $5600 \mathrm{MB}$ \\ Eindhoven, The \\ Netherlands \\ E-mail: menno.prins@philips.com
}

\begin{abstract}
We present an optical biosensor technology that integrates the tethered particle motion technique and the magnetic tweezer technique. The goal is to quantify the three-dimensional mobility of bound particle labels and to characterize the bond between the particle and the surface. We show using a series of four different lengths of dsDNA (105 bp to $590 \mathrm{bp}$ ) that plots of the height as function of the in-plane particle position reflect the bond length and bond flexibility. We analyze ensembles of bound particles and show that the height displacement is at maximum the bond length, but that non-specific sticking causes large variations between particles. We also measured the height of bound particles under influence of magnetic forces. A magnetic gradient force towards the surface brought particles on average closer to the surface, but a magnetic gradient force away from the surface did not bring all particles away from the surface. We show that the latter can be explained by magnetic anisotropy in the particles. Our results demonstrate that mobility detection of bound particle labels in an evanescent field is a promising technique to characterize the bond between a particle and a surface in a biosensor system.
\end{abstract}

\section{Short title}

Bond Characterization in Evanescent Field Biosensor

PACS

87.14.gk

87.64.M-

87.80.Nj

87.85.fk

Submitted to

Journal of Physics D: Applied Physics 


\section{Introduction}

The use of particle labels that can bind to individual molecules has enabled single-molecule sensitivity in the fields of biosensing and biophysics. In biosensors the presence of a particle label is detected to determine the concentration of a certain analyte in a body fluid. In the field of biophysics, particle labels are used to characterize the properties of individual molecules. The discovery of optical tweezers in the late 1980s and magnetic tweezers in the 1990s opened the possibility to exert well-defined forces on particles and thus on single molecules.[1-4] The introduction of the tethered particle motion technique in 1991 enabled characterization of individual molecules without applied forces.[5] By measuring the two-dimensional Brownian motion of tethered particles, information is obtained on the length and/or elasticity of individual molecules. These single-molecule techniques have led to novel understandings in fields such as protein folding, DNA transcription, DNA replication, molecular motors and enzyme activity.[6, 7]

In this paper we integrate the magnetic tweezer and tethered particle motion techniques in a biosensor system, and we measure the particle mobility in three dimensions with nanometre resolution by using an optical evanescent field.[8-11] We will show that the combination of these three techniques enables the characterization of the bond between a particle and a biosensor surface. As a model analyte we use double stranded DNA with lengths between $105 \mathrm{bp}$ and $590 \mathrm{bp}$ (36 nm to 201 $\mathrm{nm}$ ), in order to get bond lengths on the order of the evanescent field depth. We will first show that the three-dimensional mobility of bound polystyrene particles gives information on the length and flexibility of the bond, and we study the variations in ensembles of bound particles. Next, we compare the use of polystyrene particles to the use of magnetic particles. Finally, we demonstrate how the height and height mobility of bound magnetic particles can be influenced using magnetic forces towards or away from the surface, and we present a model and experimental proof of the influence of magnetic anisotropy and particle orientation on the particle height and particle mobility.

\section{Materials and methods}

\subsection{Set-up}

The evanescent wave set-up with scattered light imaging is sketched in figure 1. A light beam is coupled into a disposable, injection moulded, polystyrene cartridge $(12 \times 40 \mathrm{~mm})$ with an angle of incidence of $70^{\circ}$, using optical entrance facets perpendicular to the light beam.[12] At the sensor area the cartridge thickness is $0.8 \mathrm{~mm}$. A $2 \mathrm{~mm}$ wide fluid channel is created with $180 \mu \mathrm{m}$ thick double sided tape and a $150 \mu \mathrm{m}$ thick microscope cover glass. The light source is a red laser diode $(658 \mathrm{~nm}$, Sanyo DL-6147-240, $20 \mathrm{~mW}$ ) that is slightly focused to an optical spot of approximately $200 \mu \mathrm{m}$ in diameter. The penetration depth $d_{p}$ of the evanescent field is given by:[10]

$$
d_{p}=\left[4 \pi / \lambda \sqrt{\left(n_{1} \sin \theta_{i}\right)^{2}-n_{2}^{2}}\right]^{-1},
$$

with $\lambda$ the wavelength of the light, $n_{1}$ the refractive index of the cartridge, $n_{2}$ the refractive index of the fluid, and $\theta_{i}$ the angle of incidence of the light beam. Typical values in our set-up are a wavelength $\lambda=658 \mathrm{~nm}$, refractive indices $n_{1}=1.5, n_{2}=1.3$ and an angle of incidence $\theta_{i}=70^{\circ}$, leading to an evanescent field depth $d_{p}$ of $95 \mathrm{~nm}$. 


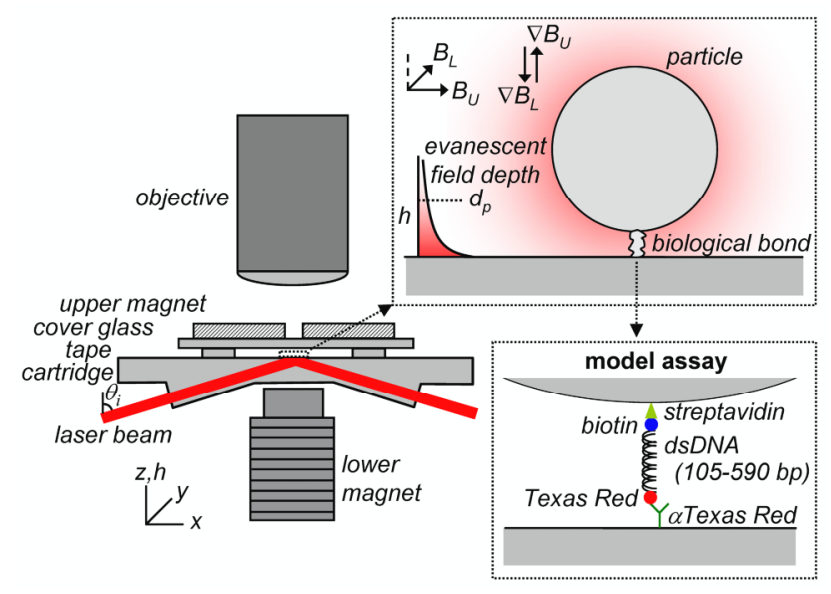

Figure 1. Biosensor set-up to detect the scattered light of particles present in the evanescent field above the sensor surface, which is generated by a laser beam coupled into the cartridge under an angle $\theta_{i}$ of total internal reflection. The scattered light intensity of the particles depends on the particle height above the surface. As a model analyte to biologically bind particles to the sensor surface we use dsDNA of four different lengths (105 bp to $590 \mathrm{bp}$ ). A lower magnet (electromagnet), with field $\mathbf{B}_{\mathbf{L}}=B_{L} \hat{y}$ and gradient $\nabla \mathbf{B}_{\mathbf{L}}=-B_{L} \hat{z}$, and an upper magnet (two permanent magnets), with field $\mathbf{B}_{\mathbf{U}}=B_{U} \hat{x}$ and gradient $\nabla \mathbf{B}_{\mathbf{U}}=B_{U} \hat{z}$, are used to magnetically manipulate bound magnetic particles.

As particles we use both streptavidin coated polystyrene particles of Spherotech Inc. with a diameter of $0.7 \mu \mathrm{m}$ (mean diameter determined from Scanning Electron Microscopy images of 760 $\mathrm{nm}$ and a coefficient of variation $(\mathrm{CV})$ of $5 \%$ ), and streptavidin coated superparamagnetic particles of MagSense with a diameter of $500 \mathrm{~nm}$ (mean diameter $580 \mathrm{~nm}, \mathrm{CV}$ of $25 \%$ ). The scattered light of the particles has been shown to relate exponentially to the height of the particles above the surface: $[9,10]$

$$
I(h)=I_{0} e^{-h / d_{p}} \text {, }
$$

where $I_{0}$ is the intensity of the particle in contact with the surface. Because of the variations in particle size and spatial variations in laser intensity in our experiments, $I_{0}$ varied and was therefore not used to calculate the absolute particle height. By rewriting Eq. 2 to:

$$
\Delta h=d_{p} \ln I_{i} / I_{\max }, \quad \text { (3) }
$$

we determined from the intensity $I_{i}$ the height $\Delta h$ relative to the position of the highest intensity $I_{\max }$ measured for each particle during an experiment. The scattered light is imaged with single particle resolution using a Leica microscope (DMLM/LP), a 50x objective lens of NA=0.50 (N plan) and a CCD camera (Pike, Allied Vision, 30-60 fps). The field of view is $96 \mu \mathrm{m}$ by $72 \mu \mathrm{m}$ (with one pixel corresponding to $150 \mathrm{~nm}$ ), enabling detection of multiple particles simultaneously. The exposure time per recorded frame is $4 \mathrm{~ms}$ for the polystyrene particles and $1 \mathrm{~ms}$ for the magnetic particles, which is sufficient for measuring accurate height information without blurring due to particle motion.[11] The recorded movies are analyzed using a Matlab routine that recognizes the particles, determines the $x, y$ position with sub-pixel accuracy $(\sim 10 \mathrm{~nm})$ by a centre of mass analysis of the intensity profile, and calculates the total measured intensity per particle by integrating all pixels belonging to a single particle.

To apply a downward magnetic gradient force to the particles we use an electromagnet below the cartridge, which is composed of two coils operated with opposite currents, giving a field $B_{L}$ and field gradient $\nabla B_{L}$, as shown in figure 1. To apply an upward magnetic gradient force we designed a holder that contains two permanent magnets in a horizontal magnetization configuration with a $1 \mathrm{~mm}$ gap,[13] giving a field $B_{U}$ and field gradient $\nabla B_{U}$ (figure 1). During an experiment the holder can be slided onto the cartridge without changing the microscope configuration. Each of the two magnets has a size of $5 \times 5 \times 1.5 \mathrm{~mm}$, and is composed of five $5 \times 1 \times 1.5 \mathrm{~mm}$ neodymium-iron-boron magnets (www.supermagnete.de, magnetization N45). Due to the small gap size, part of the light from the 
particles is shielded by the magnets, therefore a correction factor needs to be applied to the measured light intensity, which we determined to be 1.5 .

\subsection{Assay}

As a biological model system we use four fragments of double-stranded DNA with different lengths, obtained by PCR amplification: Escherichia coli partial 23S rRNA gene (590 bp (201 nm) and $141 \mathrm{bp}$ $(48 \mathrm{~nm})$ ),[14] species-specific gene fragment for Staphylococcus aureus (105 bp (36 nm)),[15] and an antibiotic resistance gene of Salmonella $(290 \mathrm{bp}(99 \mathrm{~nm})$ ).[16] The DNA strands are tagged with Texas Red on one end and biotin on the other end;[16] the particles have a streptavidin coating and the cartridge surface is coated with polyclonal antibodies against Texas red.[11] After coating the cartridge, the surface is blocked in a PBS buffer containing 1\% (w/v) BSA, 10\% (w/v) sucrose and $0.1 \%(\mathrm{w} / \mathrm{v})$ sodium azide and stored at $4^{\circ} \mathrm{C}$. Before use, the cartridge is washed with $100 \mathrm{mM}$ borate buffer ( $\mathrm{pH} 8.5$ ) containing $0.05 \%(\mathrm{v} / \mathrm{v})$ Tween 20. Both the DNA and the particles are diluted in a 100 $\mathrm{mM}$ borate buffer containing $1 \%(\mathrm{w} / \mathrm{v})$ BSA and $0.05 \%(\mathrm{v} / \mathrm{v})$ Tween 20 .

An assay is performed by adding the particle solution to the DNA solution, and incubating for 1 minute. The cartridge channel is then filled with $4 \mu \mathrm{l}$ fluid and incubated for a variable time. Finally, unbound particles are washed away using a pipette with $400 \mu 100 \mathrm{mM}$ borate buffer containing $0.05 \%(\mathrm{v} / \mathrm{v})$ Tween 20 . For the polystyrene particles, the particle concentration was $0.25 \%(\mathrm{w} / \mathrm{v})$ and the DNA concentration was $5 \mathrm{pM}$. The incubation time was 3 minutes for $590 \mathrm{bp}$ DNA, 3 minutes for 290 bp DNA, 6 minutes for 141 bp DNA and 8 minutes for 105 bp DNA. For the magnetic particles, the particle concentration was $0.1 \%(\mathrm{w} / \mathrm{v})$ and the DNA concentration $5 \mathrm{pM}$. The incubation time was 2 minutes for $590 \mathrm{bp}$ DNA, 2 minutes for $290 \mathrm{bp}$ DNA, 4 minutes for $141 \mathrm{bp}$ DNA and 5 minutes for 105 bp DNA. The incubation times were experimentally tuned to obtain a similar low density $(\sim 0.01$ particles $/ \mu \mathrm{m}^{2}$ ) of bound particles on the sensor surface. The DNA concentrations were selected to give slightly less DNA molecules than particles in the fluid (ratio 1:3 for polystyrene particles, ratio 1:2 for magnetic particles), in order to favor the formation of single molecular bonds between particles and sensor surface.

\subsection{Geometric description}

Figure 2 shows a number of parameters introduced to describe the movement of the particle and the tether. The angle between the tether and the surface is defined as $\alpha$, the angle between the tether and the particle is defined as $\beta$, the particle radius is $r$ and the length of the tether is $L$. Three idealized situations are sketched for a displacement of the particle, depending on the properties of the tether and attachment points. Situation 1 shows uniform bending of the tether over an angle $\phi$, while $\alpha$ and $\beta$ both stay $90^{\circ}$. In this case the particle height $h_{1}$ is given by:

$$
h_{1}=L \frac{\sin \phi}{\phi}+r(\cos \phi-1),
$$

and the lateral displacement $d_{1}$ by:

$$
d_{1}=L \frac{1-\cos \phi}{\phi}+r \sin \phi .
$$

In situation 2 the angle $\alpha$ between the tether and the surface changes, while $\beta$ stays $90^{\circ}$, giving a particle height $h_{2}$ of:

$$
h_{2}=(L+r) \sin (\alpha)-r,
$$

and a lateral displacement $d_{2}$ of:

$$
d_{2}=(L+r) \cos (\alpha) .
$$

In situation 3 the angle $\beta$ between the tether and the particle changes, while $\alpha$ stays $90^{\circ}$, giving a particle height $h_{3}$ of:

$$
h_{3}=L-r(1-\sin (\beta)),
$$

and a lateral displacement $d_{3}$ of:

$$
d_{3}=r \cos (\beta) .
$$




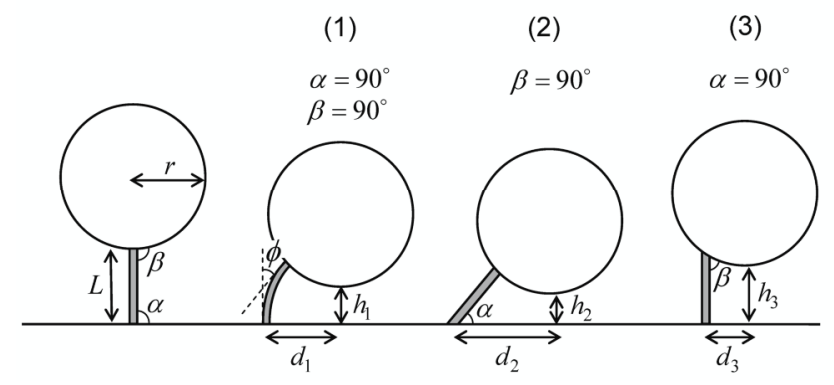

Figure 2. Definition of parameters to describe the behaviour of a particle with radius $r$ bound to the surface via a tether of length $L . \alpha$ is the angle between the tether and the surface, $\beta$ is the angle between the tether and the particle and $\phi$ is the angle of curvature of the tether. Sketch (1), (2) and (3) represent idealized possibilities for particle displacement, depending on the properties of the tether and attachment points. $h_{1}, h_{2}$ and $h_{3}$ denote the particle height for the different possibilities, and $d_{1}, d_{2}$ and $d_{3}$ denote the lateral displacement.

\section{Results and discussion}

\subsection{Particle mobility and bond flexibility}

To investigate the possibility to obtain information on the bond from the three-dimensional particle mobility, we first studied the mobility of several individual polystyrene particles, bound to the sensor surface by one of the four different DNA lengths. We measured the intensity fluctuations of the bound particles over a time period of about 30 seconds. From the intensity data we calculated (Eq. 3) the height $\Delta h$ relative to the position of highest intensity, i.e. the position of closest approach to the surface. In figure 3 we plotted for four particles that were each bound to the surface by DNA with a different length, the calculated height as a function of the in-plane position. For each particle three different colour plots are shown: the maximum height at each position, the average height at each position and the minimum height at each position. In general, figure 3 shows that the particles move in a circle with increasing radius from $(170 \pm 5) \mathrm{nm}$ to $(400 \pm 5) \mathrm{nm}$ for increasing tether length. For the shortest DNA length the maximum lateral displacements for the different situations in figure 2 are all on the right order: situation 1 gives $167 \mathrm{~nm}$, situation 2 gives $169 \mathrm{~nm}$ and situation 3 gives $161 \mathrm{~nm}$ (calculated using respectively Eq. 5 with $\phi=24.8^{\circ}$, Eq. 7 with $\alpha=66^{\circ}$ and Eq. 9 with $\beta=65^{\circ}$ ). For the longest DNA length, situation 1 gives $408 \mathrm{~nm}$, situation 2 gives $440 \mathrm{~nm}$ and situation 3 gives $335 \mathrm{~nm}$ (calculated using respectively Eq. 5 with $\phi=56.5^{\circ}$, Eq. 7 with $\alpha=40.8^{\circ}$ and Eq. 9 with $\beta=28.1^{\circ}$ ). In this case situation 1 best matches the measured radius of $(400 \pm 5) \mathrm{nm}$.

The first column in figure 3 shows that the largest maximum height is found in the centre of the circle and the lowest height is found at the contour, which is expected based on geometry (see figure 2). The maximum height is seen to increase with the tether length (note that the colour scales in the different rows vary). The second column in figure 3 shows that the average height is lower than the maximum height, demonstrating the influence of the tether flexibility. Moreover, it can be seen that the difference between the average height and the maximum height becomes larger for increasing tether length, up to a factor of two difference for the longest DNA, showing an increasing flexibility for increasing tether length. The last column in figure 3 shows that the minimum height is also largest at the centre of the circle, but this effect reduces for longer tethers lengths with increasing flexibility. 


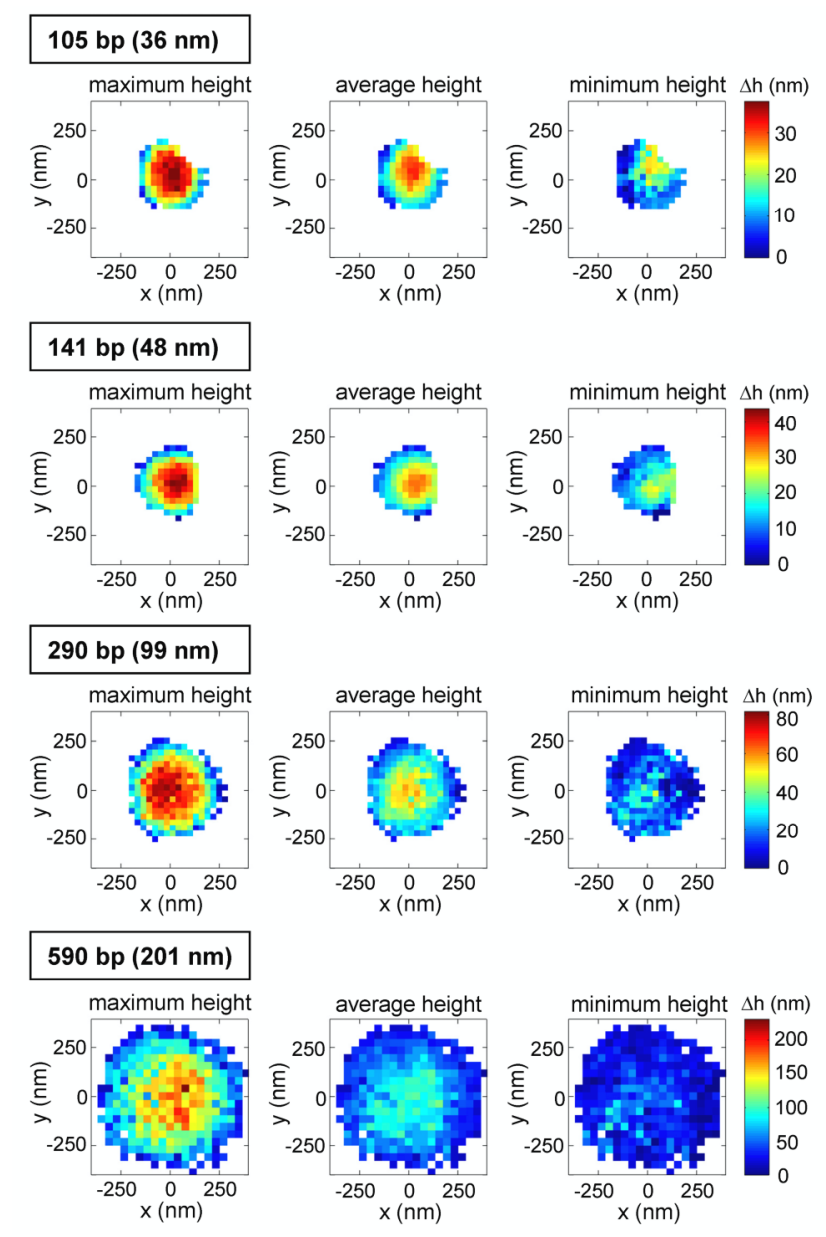

Figure 3. Relative height $\Delta h$ with respect to the position of closest approach to the surface (Eq. 3) for four $0.7 \mu \mathrm{m}$ polystyrene particles, each bound to a different DNA length. The first column shows the maximum relative height as function of the in-plane $(x, y)$ position, the second column the average relative height as function of the in-plane position and the third column the minimum relative height as function of the in-plane position, calculated (Eq. 3) from a 30 seconds intensity measurement.

The maximum relative heights in figure 3 are on the order of the DNA length, so the particle apparently easily touches the surface when moving to the contour of the circle. Because the particle is relatively large compared to the DNA length, only small angles are required (see figure 2). Taking for example the situation where the DNA bends uniformly (Eq. 4, situation 1 in figure 2), the maximum required bending angle $\phi$ ranges from $25^{\circ}$ for $36 \mathrm{~nm}$ DNA to $57^{\circ}$ for $201 \mathrm{~nm}$ DNA. DNA is known to be a semi-flexible polymer with a characteristic bending length scale that is called the persistence length. The energy to uniformly bend a strand of DNA with length $L$ over an angle $\phi$ is given by:[17]

$$
E=\frac{k_{B} T P}{2 L} \phi^{2},
$$

with $k_{B} T$ the thermal energy and $P$ the persistence length. The value of the persistence length of DNA depends on buffer conditions and temperature, but is usually taken on the order of $50 \mathrm{~nm}$.[17] For the maximum bending angles calculated above, the bending energies are below $0.14 k_{B} T$, confirming that the particle can touch the surface when moving to the contour of the circle.

For a particle to decrease its height while staying at the centre position, the bending angle $\phi$ of the DNA has to become either much larger or non-uniform, or the tether needs to pivot around one of the attachment points (angles $\alpha$ and $\beta$ ), or combinations of the three situations (figure 2) are required. In figure 3 we see for example that for the shortest DNA $(36 \mathrm{~nm})$ the minimum height at the centre position of the circle is around $20 \mathrm{~nm}$. In order to reach this height while keeping $\alpha=\beta=90^{\circ}$, the tether needs to bend more than 180 degrees, leading to estimated energies of more than $6 k_{B} T$, giving 
a negligible occurrence probability. The tether thus has some freedom to pivot around the attachment points (antibody-Texas Red or biotin-streptavidin), which is also often assumed in tethered particle motion modelling.[18, 19] For longer DNA, bending becomes increasingly easier, for example 1.2 $k_{B} T$ for bending $201 \mathrm{~nm}$ DNA over $180^{\circ}$. Indeed figure 2 shows that the relative difference between maximum and minimum height becomes larger for increasing DNA length; for the longest DNA of $201 \mathrm{~nm}$ the minimum height at centre position is around $50 \mathrm{~nm}$.

\subsection{Mobility differences between particles}

For biosensing applications, it is important to obtain detailed information on individual particles and molecules, as well as to obtain good statistics on multiple single particles and molecules. Therefore, we determined the maximum height displacement for ensembles of polystyrene particles and for ensembles of magnetic particles, in separate experiments with the four different DNA lengths.

In DNA assays with polystyrene particles, we saw that about half of the particles were immobile on the surface, showing both no in-plane position variations and no intensity variations. In control assays without DNA, we observed that only very few particles were bound to the surface, much less than in assays with DNA. This gives two possibilities for the immobile particles in the DNA assay: either the particles were specifically bound to the surface by DNA and additionally non-specifically bound to the surface, or the DNA was non-specifically bound in between the particle and the surface. In the experiments with DNA, we saw some examples of particles with normal intensity-position plots, such as in figure 3, that would sometimes temporarily stick to the surface at a position on the contour of the circle. Such observations point to the occurrence of additional non-specific sticking of specifically bound particles, which has also been seen in tethered particle motion studies.[18] To derive information on the molecular bond between particle and surface, we focused our analyses on particles that are bound and also show in-plane mobility.

For the particles with in-plane movements, histograms of the measured maximum heights were made for each DNA length, see figure $4(a)$. The vertical black lines denote the DNA length. The dashed vertical lines give a possible addition of $15 \mathrm{~nm}$ due to the antibody and linkers (antibody is approximately $15 \mathrm{~nm},[20]$ streptavidin approximately $5 \mathrm{~nm}$, and biotin and texas red are on the nanometre scale). It can be seen that the found maximum heights are generally below the possible values due to the bond length, and that there is a large variation between particles, with a random distribution from zero to the maximum expected value. Also, a few cases are seen where the derived maximum height is higher than the bond length.

We also measured the maximum height displacements for ensembles of MagSense magnetic particles and the four different DNA lengths. We found that for the magnetic particles there was much less sticking to the surface than for the polystyrene particles. Figure $4(b)$ shows for each DNA length a histogram of the found maximum heights of all particles, with the only selection being that particles should be far enough away from each other to prevent overlapping optical profiles. It can be seen that there is still a large variation in found heights between particles, but the peak of the distribution clearly shifts with the DNA length. Also, particles are found with a maximum height that is larger than to be expected from the tether length, especially for the two shortest DNA lengths. In another publication we have shown that optically non-uniform magnetic particles (i.e. non-spherical, rough surface and inhomogeneous content) can show an increase in scattering intensity fluctuations, thus leading to an overestimation of the height displacement.[11] MagSense particles are observed in Transmission Electron Microscopy images to be smooth and spherical, but some particles may still have minor defects or an inhomogeneous content, leading to a small increase in measured height, which becomes noticeable for short tether lengths. 
(a)

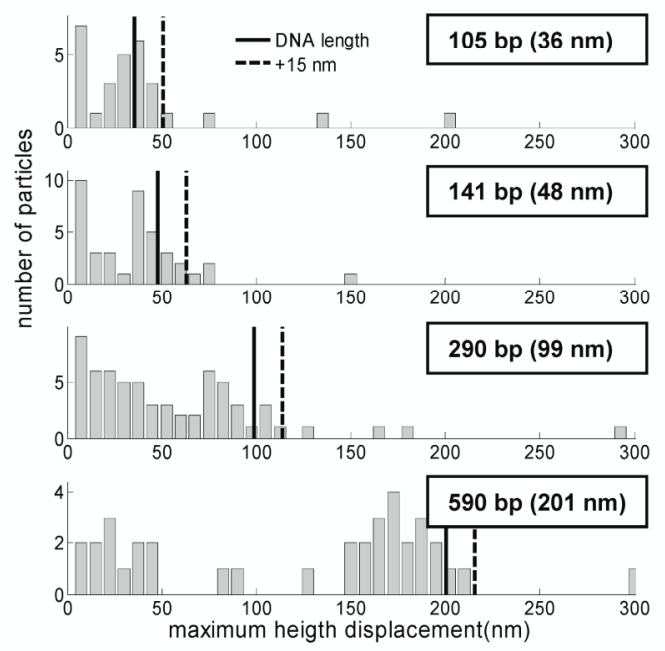

(b)

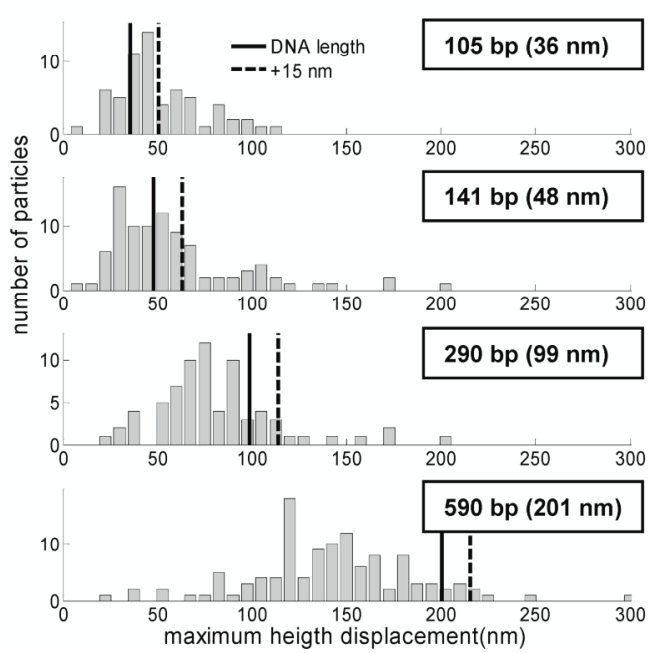

Figure 4. (a) Histograms of the measured maximum height displacements of ensembles of polystyrene particles bound to the surface via the four different DNA lengths. The black vertical lines in the graphs denote the DNA length, the dashed lines the DNA length plus an estimated maximum extra length of $15 \mathrm{~nm}$ due to antibody and linker molecules. (b) Histograms of the measured maximum height displacements of ensembles of magnetic particles bound to the surface via the four different DNA lengths.

We examined the occurrence of heights smaller than the tether length for both particle types by studying the intensity-position plots, and found many differences in mobility between different cases. For example, we observed particles with normal $x, y$-displacement and reduced height, particles with reduced $x, y$-displacement and reduced height, or particles with asymmetric $x, y$-displacement (such as half-spherical plots). A possible cause for reduced heights may be the occurrence of double bonds. We estimate using Poisson statistics [21], taking account of the distance between molecules on the particle surface, and assuming full flexibility of the attachment points, that indeed part of the cases may be caused by double bonds. For the specifically bound polystyrene particles, at maximum $15 \%$ can have double bonds for $36 \mathrm{~nm}$ DNA, and at maximum $27 \%$ for $201 \mathrm{~nm}$ DNA. For the specifically bound magnetic particles, at maximum $34 \%$ can have double bonds for $36 \mathrm{~nm}$ DNA, and at maximum $54 \%$ for $201 \mathrm{~nm}$ DNA. The remaining reduced-height cases may be caused by various ways of additional non-specific binding of specifically bound particles, such as that part of the DNA strand is sticking to the surface, giving a lower height but also less freedom to move in circular symmetry. A final possibility is that especially for longer DNA tethers additional energy is needed to completely stretch the bond, as the statistical probability of finding a long flexible string fully stretched becomes smaller.[22] An estimation based on the worm-like chain model, proven to be applicable to DNA longer than $1 \mu \mathrm{m}$,[23] would give for $201 \mathrm{~nm}$ DNA an energy over $5 k_{B} T$ to stretch the effective length over $160 \mathrm{~nm}$. This energy is, however, larger than observed for many particles in our experiments.

In order to distinguish in a biosensor single bonds with different lengths by measuring the in-plane particle displacement or the maximum height displacement, additional optimization is thus needed, such as further reducing the possibility for double bonds and reducing non-specific sticking. The latter was less for the magnetic particles than for the polystyrene particles in the same assay conditions, showing the importance of tuning the surface properties of the particles. Additional blocking agents or different buffer conditions, e.g. different ionic strength or different $\mathrm{pH}$, may further improve the assay. Another possibility is to manipulate the particles using applied forces, which we will investigate in the following sections.

\subsection{Influence of magnetic forces on particle mobility}

When using magnetic particles, the height distribution function can be influenced with magnetic forces. We investigated the effect of magnetic forces on the particle height using two magnetic 
systems: a lower magnet and an upper magnet, as explained in Section 2.1 and figure 1 . The lower magnet is an electromagnet which gives a field up to about $50 \mathrm{mT}$ parallel to the cartridge surface (in the $y$-direction) and a field gradient force towards the surface (in the $-z$-direction). The estimated magnitude of the gradient force of the lower magnet is $0.3 \mathrm{pN}$. The upper magnet is made of permanent magnets and gives a field of about $500 \mathrm{mT}$ parallel to the cartridge surface (in the $x$ direction) and a field gradient force away from the surface (in the $+z$-direction). The estimated magnitude of the force of the upper magnet is $1 \mathrm{pN}$.

We first tested the influence of the lower magnet. Figure $5(a)$ shows the position and intensity as a function of time for a particle bound to $141 \mathrm{bp}$ DNA, when alternately turning the lower magnet on and off. It can be seen that when the magnet is turned on, the particle becomes more strongly restricted in $x, y$-position, the average intensity increases and the fluctuations in intensity decrease. It can also be seen that the particle takes two different positions in the magnetic field: one towards the $+y$-direction and towards the $-y$-direction. Figure $5(b)$ shows a scatter plot of the $x, y$-positions with the magnetic field (grey data points) and without the magnetic field (black data points). The two fieldinduced positions are at the contour of the circle of the field-free positions, and are 180 degrees apart. Finally, figure $5(c)$ shows the height distributions of the particle with and without the magnetic field. On average the particle is indeed closer to the surface when it is magnetically pulled towards the surface. There still is a considerable mobility in height of about $40 \mathrm{~nm}$ when the magnetic field is turned on. For a larger ensemble of particles we found height mobilities ranging from $20 \mathrm{~nm}$ to 120 $\mathrm{nm}$. These displacements correspond to forces between $0.1 \mathrm{pN}$ and $0.6 \mathrm{pN}$ (roughly estimated by taking the probability for a free particle of moving a certain height $\Delta z$ against a force $F$ : $\left.P \sim \exp \left(-F \Delta z / k_{B} T\right)\right)$. The range in forces is probably caused by the fact that the particle diameters vary up to a factor of two, so magnetic gradient forces may vary an order of magnitude.[24, 25]

(a)
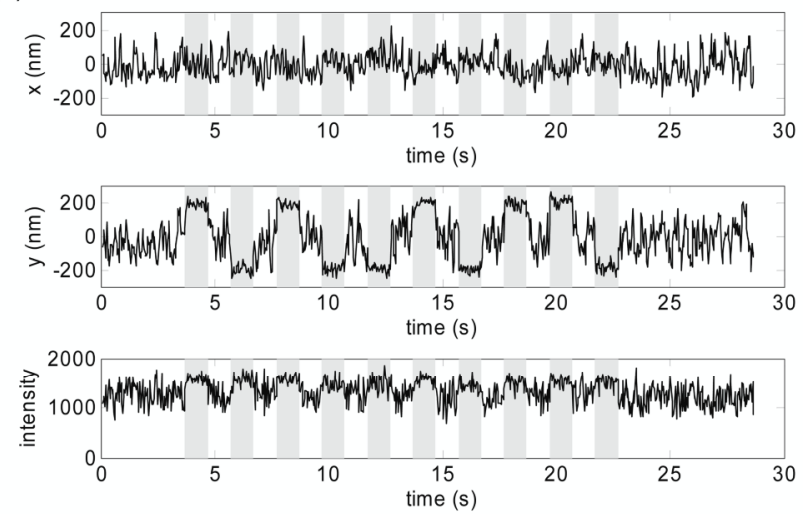

(b)

(c)
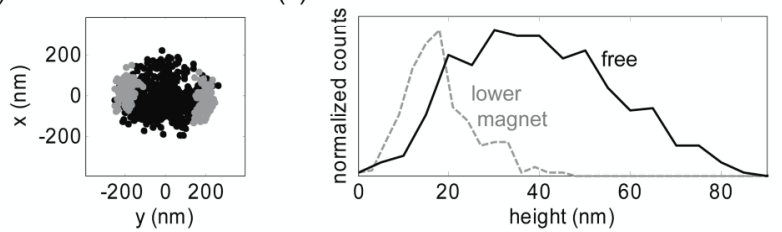

Figure 5. (a) Position and intensity versus time for a particle bound to $141 \mathrm{bp}$ DNA when alternately turning the lower magnet on (grey bars) and off (outside grey bars). (b) Scatter plot of the positions in the magnetic field (grey) and without the magnetic field (black). (c) Histogram of the height distribution in the magnetic field (dashed grey) and without the magnetic field (black).

Next, we investigated the influence of the upper magnet on the particle mobility. We found that the upper magnet strongly confines the height of the bound particles (within 5 to $30 \mathrm{~nm}$ ), corresponding to forces between 0.4 and $2.4 \mathrm{pN}$. Surprisingly, the upper magnet confined particles to different height levels: we observed that more than half of the particles were not pulled to the highest possible height. Figures $6(a)$-(c) show three examples of particles upon actuation by the upper magnet, giving a resulting height of respectively the highest possible height, a medium height and the 
lowest possible height. Such behaviour was observed for all four DNA lengths, and was reproduced when the upper magnet was removed and subsequently repositioned. When analyzing a large ensemble of magnetic particles, the relative distribution over maximum-medium-minimum heights was about 60\%-20\%-20\% for the 290 bp DNA, and 40\%-30\%-30\% for the 105 bp DNA. In the next section we will describe a possible cause for the different height levels under an applied magnetic field.

(a)

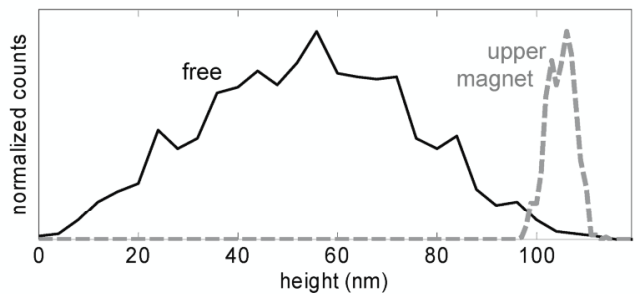

(b)

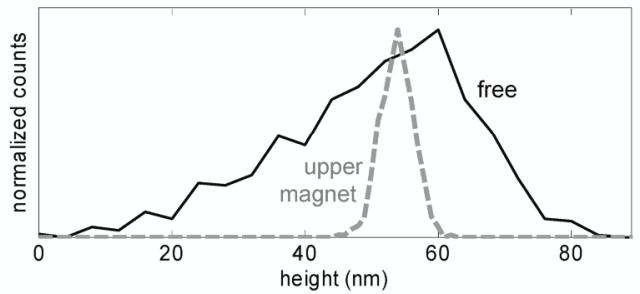

(c)

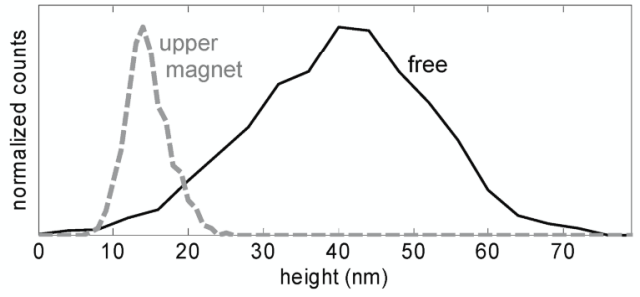

Figure 6. (a) Histogram of the height distribution of a particle bound to 290 bp DNA when applying the upper magnet (dashed grey) and without the magnet (black), showing that the particle is pulled to its maximum height. (b) Example of a situation where another bound particle (290 bp DNA) is confined at an average height using the upper magnet. (c) Example of a situation where a bound particle (290 bp DNA) is pushed towards the surface using the upper magnet.

Finally, we tested the influence of the magnetic fields on particles with reduced height displacements or asymmetric intensity-position plots. We found that fields from neither the lower magnet nor the upper magnet altered the particle behaviour irreversibly. The induced forces were thus too small to break loose possible additional non-specific binding of specifically bound particles. In a magnetic field, particles mostly assumed height values that were already available in the field-free height distribution; only occasionally $(\sim 10 \%)$ we measured in field a height slightly below or above the extreme values of the height distribution in the field-free state. Upon its placement, the top magnet did cause immediate rupturing of a fraction of the particles (10-20\%), but the chance of rupturing did not seem to be related to the intensity-position plots of the particles prior to rupturing.

\subsection{Influence of magnetic anisotropy on particle mobility}

In the previous section we described two interesting phenomena. First, we showed that when applying the lower magnet, the particle switched between two defined $x, y$-positions that were at the contour of the circle of the free positions and were 180 degrees apart. Secondly, we showed that when applying the upper magnet more than half of the particles were not pulled to the highest possible height, but were sometimes even brought towards the surface. In this section we propose a model to account for the influence of magnetic anisotropy of the particle on the field-dependent particle mobility. 
When pulling a particle towards the surface using the lower magnet, the stiffness of the tether will cause the particle to move to the contour of the circle (see figure 2). However, ideal spherical superparamagnetic particles should be able to take any position on the contour of the circle, since they immediately remagnetise when rotating. The occurrence of the two position states (figure $5(b)$ ) may therefore point to magnetic alignment of the particles. We observed the occurrence of defined position states for all particles, but the degree of confinement in $x, y$-position was dependent on the particle. Some particles showed only one recurring position, but most particles showed two positions. Moreover, the aligned positions were often mostly directed towards the direction of the field ( $y$-direction). The choice for either one of the two states was random over time, like in figure $5(a)$, and showed that the orienting behaviour was not due to an additional magnetic field gradient and thus force in the $y$-direction. We also excluded the influence of particle-particle interactions, as we calculated that forces between particles were negligible for distances larger than $6 \mu \mathrm{m}$, which was the case in the experiments.

A magnetic anisotropy in the particles can cause particle alignment in a magnetic field. Magnetic anisotropy can originate for example from shape anisotropy (non-spherical particles), from an uneven distribution of the magnetic content inside the particle or from a small permanent magnetic moment. As we observed MagSense particles in Transmission Electron Microscopy images to be smooth, spherical and homogeneous, the most probable cause for magnetic anisotropy is a small permanent moment. It has been shown for $3 \mu \mathrm{m}$ superparamagnetic particles that a small permanent moment indeed exists, with values up to $1 \%$ of the saturation magnetization.[26] The rotation energy $E$ of a magnetic particle with permanent moment $m_{p}$ in a magnetic field $B$ is given by:

$$
E=-m_{p} B \cos \theta,
$$

with $\theta$ the angle between the moment and the field. A $500 \mathrm{~nm}$ MagSense particle with a permanent moment of $0.5 \%$ of the saturation magnetization $m_{\text {sat }}\left(m_{\text {sat }}=8.9 .10^{-15} \mathrm{Am}^{2}\right)$ will align for example in a field of $50 \mathrm{mT}$ within $6^{\circ}$ (energy of $3 \mathrm{k}_{\mathrm{B}} \mathrm{T}$ ). A MagSense particle with a permanent moment of $0.1 \%$ $m_{\text {sat }}$ will align in a field of $50 \mathrm{mT}$ within $13^{\circ}$. For higher fields this angle reduces, for example to $2^{\circ}$ $4^{\circ}$ for a MagSense particle with a moment between $0.1-0.5 \% m_{\text {sat }}$ in a field of $500 \mathrm{mT}$. Alignment effects can thus well be expected in our experiments.

The magnetic anisotropy axis (i.e. permanent moment) of a bound particle will probably have a random orientation with respect to the attachment point of the DNA on the particle (figure $7(a)$, angle $\gamma$ ) and thus with respect to the magnetic field (figure $7(a)$, angle $\theta$ ), which in our case is parallel to the surface. When the anisotropy axis of the particle is not aligned to the field in the $x-y$ plane, a magnetic torque will cause the particle to rotate. Because in our experiments the particle is bound to the surface via single bonds (biotin to streptavidin and texas red to anti-texas red) and the DNA molecule has a low torsional constant, we expect that this rotation can occur almost without restriction. This assumption is also made in tethered particle motion modelling.[18, 19] However, when the anisotropy axis of the particle is not aligned in the $z-y$ plane, the torque-induced particle rotation does experience restrictions due to the tether. In this case, particle rotation gives a reduction in the maximum height of the particle, as is shown in figure $7(b)$. Thermal particle movement in the $y$-direction will be severely hindered because this requires either large changes in the attachment point angles ( $\alpha, \beta$, figure 2), strong DNA bending ( $\phi$, figure 2), or misalignment of the anisotropy axis. Thermal movement in the $x$-direction is easier because the particle can rotate around its anisotropy axis (clockwise towards $+x$ or anticlockwise towards $-x$ ) inducing only slight DNA bending ( $\phi$, figure 2) or rotation/twisting (not depicted). The resulting preferred path of particle movement is shown as a grey circle arc in figure $7(b)$. In order to explain two symmetric aligned states that are $180^{\circ}$ apart, as observed in our experiments (figure $5(b)$ ), the tether needs to rotate over $180^{\circ}$ and the particle magnetization needs to flip along the anisotropy axis (dashed anisotropy arrow in figure 7 (b)). Rotation over $180^{\circ}$ is expected to be possible (see above) and flipping of the magnetization can be expected when the coercive field is lower than the applied field. 
(a)

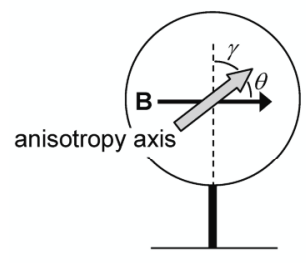

(b)

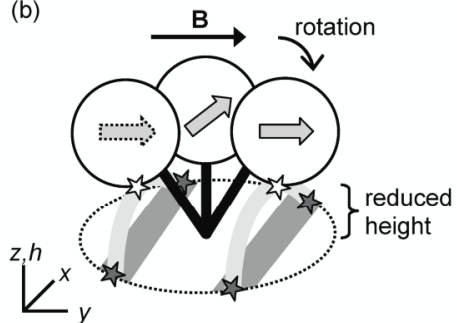

(c)

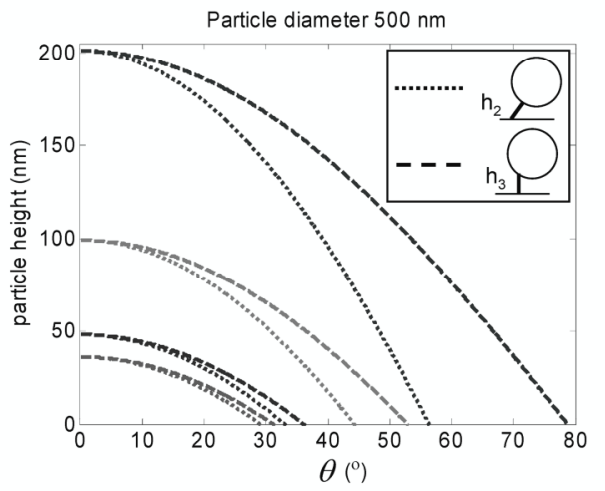

Figure 7. (a) Definition of the angle $\theta$ between the anisotropy axis of the particle and the magnetic field $\mathbf{B}$, and the angle $\gamma$ of the anisotropy axis with respect to the attachment point of the tether. (b) Schematic drawing of the particle height reduction induced when a tethered particle rotates to align its anisotropy axis with the field. The dashed arrow shows a state in which the bond is $180^{\circ}$ rotated and the particle magnetization is flipped. The two circle arcs show a path for preferred particle movement when keeping the anisotropy axis aligned. (c) Calculated maximum $\left(h_{2}\right.$, Eq. 6$)$ and minimum $\left(h_{3}\right.$, Eq. 8$)$ height reduction caused by a rotation of the particle over angle $\theta$, starting from the four different DNA lengths.

The final $x, y$-positions of the particle depend on the height reduction of the aligned state, which in turn depends on both the angle $\theta$ between the anisotropy axis and the field (figure $7(a)$ ) and the properties of the tether and attachment points. Particle rotation over the angle $\theta$ is equivalent to a rotation of either $\phi=\theta, \alpha=90^{\circ}-\theta$ or $\beta=90^{\circ}-\theta$ as explained in figure 2 . The minimum height the particle will take due to rotation is given by Eq. 6 and the maximum height is given by Eq. 8; Eq. 4 or combinations of the three situations of figure 2 will give a height in between. Figure 7 (c) shows the minimum and maximum particle height for a $500 \mathrm{~nm}$ particle as function of $\theta$, starting from the four different DNA lengths. It can be seen that for all tether lengths cases of complete height reduction (particle touching the surface) may be expected when assuming that the initial angle of the anisotropy axis can have any value between $0^{\circ}$ and $90^{\circ}$. Moreover, the shorter the tether the smaller the angle necessary for equal relative height reduction.

The different height levels observed in the magnetic field of the upper magnet, as shown in figure $6(a)-(c)$, can now be explained by a height reduction due to particle alignment. When a particle aligns in the field, the maximum height is reduced, but the particle can still move towards the surface (grey arc, figure $7(b))$. When a particle is additionally pulled upward by a magnetic gradient force, the thermal movement becomes completely restricted to the highest position on the arc, depicted as a white star in figure $7(b)$. Height reduction in spite of un upward magnetic gradient force can thus well be expected. The fact that we observed for shorter DNA (105 bp) more height reduction than for longer DNA (290 bp) can be explained by the graph of figure 7 (c), showing that for shorter DNA smaller angles are required to touch the surface. In fact, a balance exists between the energy due to the gradient force and the energy due to the rotation. When for example a particle decreases its height by $36 \mathrm{~nm}$ against a pulling force of $1 \mathrm{pN}$, a penalty of $9 k_{B} T$ is induced. We calculated (Eq. 11, $0.1 \%$ $m_{\text {sat }}, 500 \mathrm{mT}$ ) that this can be counteracted with a rotation over an angle of $8^{\circ}$. For a height decrease of $201 \mathrm{~nm}$, an energy of $50 k_{B} T$ is required, which is reached for angles larger than $17^{\circ}$. It would be interesting to analyze the position data of the pulled-up state and compare this to the free position 
distribution to gain more insight in all energies that play a role. Unfortunately we were not able to do this in our set-up, because the placement of the magnet holder on the microscope always caused a small position change of the microscope table.

We checked that the height distributions observed in the field of the upper magnet cannot be explained by a gradient force that is not exactly perpendicular to the surface. We determined by magnetic field simulations (Comsol Multiphysics) that for the dimensions in our system (gap size 1 $\mathrm{mm}$, distance magnet to surface $330 \mu \mathrm{m}$ ), the angle that the gradient force makes with the surface normal is below 10 degrees over the entire gap distance. This gives an influence on particle height of less than $10 \%$, and is thus not able to explain results as in figure 7 (c).

The behaviour of the particle in the field of the lower magnet, shown in figure $5(a)-(c)$, can be explained by a nearly complete height reduction due to particle alignment. For cases where the height reduction due to particle alignment is smaller, the particle positions in the field are expected to show more the arc-behaviour as depicted in figure $7(b)$. We indeed saw examples of this behaviour. When the height reduction is small but the downward gradient force is strong, the higher (more centred) positions on the arc become less favourable, and the particle moves towards one of the grey starred positions on the contour of the circle (figure $7(b)$ ). We observed for about $30 \%$ of the measured particles strongly confined positions on the circle arc instead of symmetric confinement with respect to the $y$-axis. We generally observed two positions $180^{\circ}$ apart, except for a few cases wherein four positions were observed (see Appendix). The occurrence of two positions instead of four might be caused by a preference for the DNA molecule to bend, rotate or twist in certain directions. We tested the effect of a decreasing field on particles that were strongly confined to two positions on the circle contour, and found that the alignment behaviour could be observed for fields down to a few $\mathrm{mT}$ (see Appendix). For fields below $18 \mathrm{mT}$ the downward gradient force was lower than $40 \mathrm{fN}$, having no measurable effect on the particle height, so the particle behaviour is then fully caused by particle alignment. For a decreasing field, the confinement changed from the two positions on the circle contour to a symmetric behaviour around the $y$-axis.

To study the influence of the field orientation on the alignment of $x, y$-positions, we investigated the behaviour of three bound particles in different fields from the lower magnet, namely by first actuating the two coils of the lower magnet in the normal way (simultaneously with opposite currents), and then by actuating one or the other coil separately. The left plots in figure $8(a)-(c)$ show the behaviour for normal actuation with the two coils. It can be seen that for particle $(a)$ the aligned positions are towards the $x$-direction, which can be explained by an anisotropy axis that is aligned to the field when the bond is stretched and oriented upward, and an additional downward force giving a preference for the positions of lower height near the contour of the circle. For particle $(b)$ and $(c)$ the aligned positions are towards the $y$-direction, which can be explained by an anisotropy axis that is not aligned to the field in the stretched bond configuration.

The middle plots in figure 8 show the aligned positions of the three particles when only one of the two coils is turned on, which gives a field that is slightly out-of-plane. The aligned positions of particle $(a)$ now shift to the left due to counter-clockwise particle rotation. Moreover, because the downward force of one coil is less strong, the particle mobility over the circle arc (figure $7(b)$ ) increases. The aligned position of particle $(b)$ can be explained by the particle rotating counterclockwise from the $+y$-aligned state of the two coils configuration, shifting its position towards the middle. The aligned positions of particle $(c)$ can be explained by the particle being prevented by the surface from rotating further counter-clockwise from the $-y$-aligned state of the two coils configuration. The right plots in figure 8 show the aligned positions of the three particles when the other coil is turned on, giving a field slightly out-of-plane in the other direction. Particle $(a)$ then shifts slightly to the right due to clockwise rotation, particle $(b)$ shifts completely to the right due to clockwise rotation and particle $(c)$ shifts to the middle due to clockwise rotation. All observations of figure 8 can thus indeed be explained by the alignment model of figure 7 . 

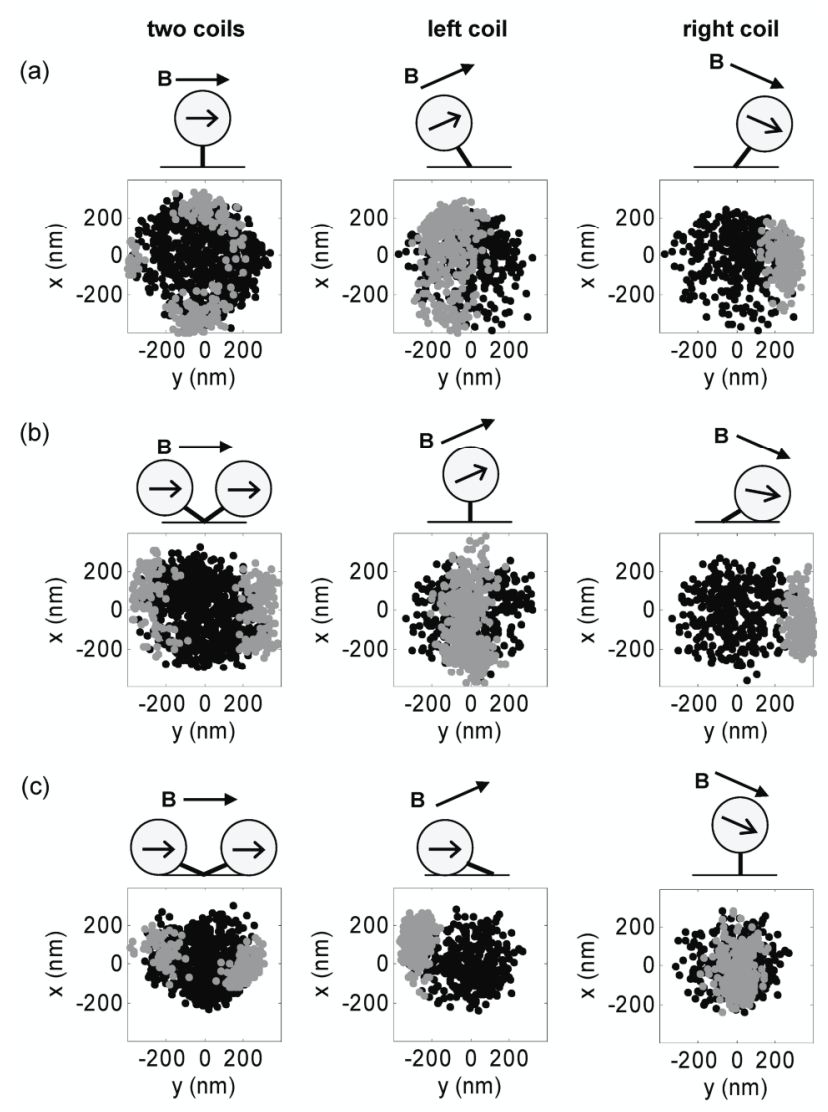

Figure 8. Scatter plots of the positions of three particles $(a),(b)$ and $(c)$, all bound to $590 \mathrm{bp} \mathrm{DNA}$, measured simultaneously in one experiment with the lower magnet turned off (black) and on (grey). For each particle the left plot shows the behaviour when the two coils of the lower magnet are actuated simultaneously (normal actuation), the middle plot shows the behaviour when only the left coil is actuated and the right plot shows the behaviour when only the right coil is actuated. The drawings show the probable orientation of the anisotropy axis of the particle with respect to the tether attachment point and the applied magnetic field, derived from the measured behaviour and the alignment model.

Summarizing, we have shown that in an evanescent-field biosensor one can measure changes in height of a bound particle under influence of magnetic gradient forces towards or away from the surface, and we have demonstrated that magnetic particle alignment and the flexibility of the biological molecules have an influence on the height of the particle. The results show that the measurement of particle height-position plots as a function of magnetic field allows a detailed probing of the properties of the magnetic particles and of the rotation and flexibility of the biological bonds. For future work it will be interesting to design electromagnets with higher field gradients and low fields, in order to favor gradient forces with respect to alignment forces. Alternatively, one can exploit the alignment behaviour by designing magnetic systems in which the field orientation can accurately be manipulated.

\section{Conclusions}

We measured the three-dimensional mobility of bound particle labels in an optical biosensor using the techniques of tethered particle motion and evanescent field detection. As a model assay we used dsDNA of four different lengths (590 bp/201 nm, $290 \mathrm{bp} / 99 \mathrm{~nm}, 141 \mathrm{bp} / 48 \mathrm{~nm}$ and $105 \mathrm{bp} / 36 \mathrm{~nm}$ ). We showed that by plotting the minimum height, the average height and the maximum height versus in-plane position, detailed information can be obtained on the length and flexibility of the bond. Next, we showed that for ensembles of individual polystyrene particles the height displacement was at maximum the expected value based on the DNA length plus additional antibodies and linkers. There was a considerable binding variability among particles due to additional non-specific sticking of 
specifically bound particles and the occurrence of double bonds. The results were similar for ensembles of magnetic particles, except for a small influence of non-uniform optical properties leading to a slight overestimation of the height displacement. When using magnetic particles, the mobility in height could be influenced by magnetic forces. A magnetic gradient force towards the surface brought particles on average closer to the surface. However, a magnetic gradient force away from the surface did not bring all particles away from the surface, as about half of the particles took medium heights or were pulled to the surface. We described a model that illustrates how magnetic anisotropy bring a bound particle into defined three-dimensional positions, and showed that this model can explain both the varying up- or downward movements in the pulling-up experiments and the alignment behaviour in the pulling-down experiments. Our results show that mobility detection of bound particle labels in an evanescent field is a promising technique to characterize the bond between a particle and a surface in a biosensor system, and that the use of magnetic fields and magnetic forces offers a rich toolbox for additional manipulation and characterization.

\section{Acknowledgments}

This work was partially funded by the NanoNed program of the Dutch Ministry of Economic Affairs.

\section{Appendix: Particle alignment in field of lower magnet}

Figure A1 shows the behaviour of a strongly orienting, bound particle in the field of the lower magnet, for different field strengths. For fields below $9 \mathrm{mT}$ the downward gradient force is negligible (lower than $10 \mathrm{fN}$ ). It can be seen that alignment can be observed for fields down to $2 \mathrm{mT}$. The position plots slowly change from two positions $180^{\circ}$ apart (grey starred positions on the circle contour, figure $7(c)$ ) to a more symmetric configuration around the $y$-axis. Note that for the highest field in figure $\mathrm{A} 1$, the maximum $x, y$-displacement away from the centre of the circle is larger than in the free state. This shows that under influence of magnetic forces and torques, the tether behaviour becomes different, changing for example the degree to which the tether can pivot around the attachment points ( $\alpha$ and $\beta$, figure 2). We also saw some cases where the degree of confinement and the measured height for the two positions could differ slightly. This has to be investigated further.

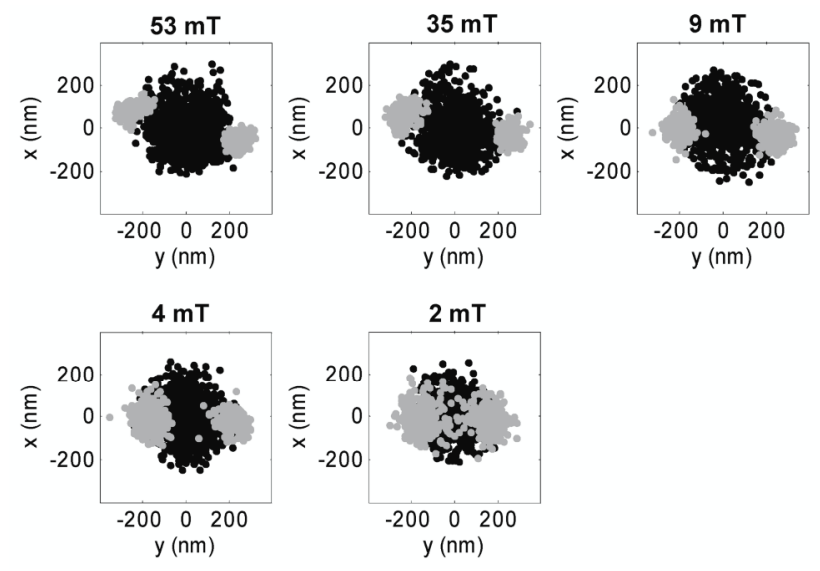

Figure A1. Scatter plots of the positions of a magnetic particle bound to 290 bp DNA in the magnetic field (grey) and without the magnetic field (black), for decreasing field strength.

Figure A2 shows a few cases where we did not observe two aligned positions that were $180^{\circ}$ apart and symmetric around $x=0$, but four aligned positions or two aligned positions symmetric around $y=0$. These cases may be explained by non-specific binding, leading to different bending and rotation behaviour of the tethered particle. 


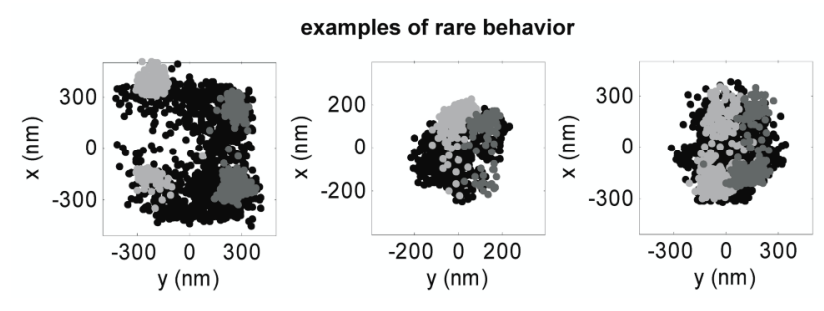

Figure A2. Three examples of rare particle behaviour, which do not show two aligned positions $180^{\circ}$ apart, but either four aligned positions or two aligned positions symmetric around $\mathrm{y}=0$.

\section{References}

[1] Ashkin A, Dziedzic J M, Bjorkholm J E and Chu S 1986 Observation of a single-beam gradient force optical trap for dielectric particles Opt. Lett. 11 288-90

[2] Block S M, Goldstein L S B and Schnapp B J 1990 Bead movement by single kinesin molecules studied with optical tweezers Nature 348 348-52

[3] Smith S B, Finzi L and Bustamante C 1992 Direct mechanical measurements of the elasticity of single DNA molecules by using magnetic beads Science 258 1122-6

[4] Strick T R, Allemand J-F, Bensimon D and Croquette V 1998 Behavior of supercoiled DNA Biophys. J. 74 2016-28

[5] Schafer D A, Gelles J, Sheetz M P and Landick R 1991 Transcription by single molecules of RNA polymerase observed by light microscopy Nature 352 444-8

[6] Deniz A A, Mukhopadhyay S and Lemke E A 2008 Single-molecule biophysics: at the interface of biology, physics and chemistry J. R. Soc. Interface 5 15-45

[7] Kapanidis A N and Strick T 2009 Biology, one molecule at a time Trends Biochem. Sci. 34 234-43

[8] Blumberg S, Gajraj A, Pennington M W and Meiners J-C 2005 Three-dimensional characterization of tethered microspheres by total internal reflection fluorescence microscopy Biophys. J. 89 1272-81

[9] Prieve D C 1999 Measurement of colloidal forces with TIRM Adv. Colloid Interface Sci. 82 93-125

[10]Prieve D C, Luo F and Lanni F 1987 Brownian motion of a hydrosol particle in a colloidal force field Faraday Discuss. Chem. Soc. $\mathbf{8 3} 297-307$

[11]van Ommering K, Somers P A, Koets M, Schleipen J J H B, van IJzendoorn L J and Prins M W J 2010 Mobility and height detection of particle labels in an optical evanescent wave biosensor with single-label resolution J. Phys. D : Appl. Phys. 43155501

[12]Bruls D M, Evers T H, Kahlman J A H, van Lankvelt P J W, Ovsyanko M, Pelssers E G M, Schleipen J J H B, de Theije F K, Verschuren C A, van der Wijk T, van Zon J B A, Dittmer W U, Immink A H J, Nieuwenhuis J H and Prins M W J 2009 Rapid integrated biosensor for multiplexed immunoassays based on actuated magnetic nanoparticles Lab Chip 93504

[13]Lipfert J, Hao X and Dekker N H 2009 Quantitative modeling and optimization of magnetic tweezers Biophys. J. 96 5040-9

[14]Mitterer G, Huber M, Leidinger E, Kirisits C, Lubitz W, Mueller M W and Schmidt W M 2004 MicroarrayBased Identification of Bacteria in Clinical Samples by Solid-Phase PCR Amplification of 23S Ribosomal DNA Sequences J. Clin. Microbiol. 42 1048-57

[15]Martineau F, Picard F J, Roy P H, Ouellette M and Bergeron M G 1998 Species-Specific and UbiquitousDNA-Based Assays for Rapid Identification of Staphylococcus aureus J. Clin. Microbiol. 36 618-23

[16]Koets M, van der Wijk T, van Eemeren J T W M, van Amerongen A and Prins M W J 2009 Rapid DNA multi-analyte immunoassay on a magneto-resistance biosensor Biosens. Bioelectron. 24 1893-8

[17]Brinkers S, Dietrich H R C, de Groote F H, Young I T and Rieger B 2009 The persistence length of double stranded DNA determined using dark field tethered particle motion J. Chem. Phys. 130215105

[18]Nelson P C, Zurla C, Brogioli D, Beausang J F, Finzi L and Dunlap D 2006 Tethered particle motion as a diagnostic of DNA tether length J. Phys. Chem. B $11017260-7$

[19]Segall D, Nelson P and Phillips R 2006 Volume-Exclusion Effects in Tethered-Particle Experiments: Bead Size Matters Phys. Rev. Lett. 96088306

[20]Giacomelli C E 2006 Adsorption of Immunoglobulins at Solid-Liquid Interfaces Encyclopedia of Surface and Colloid Science $2^{\text {nd }}$ edition, ed Somasundaran P (Boca Raton, FL: Taylor and Francis Group) p 518

[21]Osborne M A, Furey W S, Klenerman D and Balasubramanian S 2000 Single-molecule analysis of DNA immobilized on microspheres Anal. Chem. 2000, 3678-81

[22]Bustamante C, Bryant Z and Smith S B 2003 Ten years of tension: single-molecule DNA mechanics Nature 421 423-7 
[23]Kruithof M, Chien F, de Jager M and van Noort J 2008 Subpiconewton dynamic force spectroscopy using magnetic tweezers Biophys. J. 94 2343-8

[24]van Ommering K, Lamers C C H, Nieuwenhuis J H, van IJzendoorn L J and Prins M W J 2009 Analysis of individual magnetic particle motion near a chip surface J. Appl. Phys. 105104905

[25]van Ommering K, Nieuwenhuis J H, van IJzendoorn L J, Koopmans B and Prins M W J 2006 Confined Brownian motion of individual magnetic nanoparticles on a chip: Characterization of magnetic susceptibility Appl. Phys. Lett. 89142511

[26]Janssen X J A, Schellekens A J, van Ommering K, van Ijzendoorn L J and Prins M W J 2009 Controlled torque on superparamagnetic beads for functional biosensors Biosens. Bioelectron. 24 1937-41 